\title{
Neuroendocrine correlates of childhood trauma in CFS
}

Filip Van Den Eede and Greta Moorkens

We read the Review article written by Andrew S. Papadopoulos and Anthony J. Cleare (Papadopoulos, A. S. \& Cleare, A. J. Hypothalamic-pituitary-adrenal axis dysfunction in chronic fatigue syndrome. Nat. Rev. Endocrinol. 8, 22-32; 2012) ${ }^{1}$ with a lot of interest. Although we generally agree with their point of view, we would like to comment on the following inaccuracies made by the authors when referring to the results of a study performed by our research group. ${ }^{2}$

In Table 3, the authors incorrectly mention that patients with chronic fatigue syndrome (CFS) with early-life stress showed lower cortisol responses than both controls and patients with CFS without early-life stress, after dexamethasone and after the combination with corticotropinreleasing hormone. On the contrary, it was the group without early-life stress that showed significantly lower cortisol responses than the other two groups. ${ }^{2}$ Furthermore, it is not correct to state that the "mean saliva cortisol levels of those [CFS patients] without early-life stress as well as those with early-life stress were significantly lower [after $0.5 \mathrm{mg}$ dexamethasone] than those of healthy controls" (page 26), nor that the cortisol output in the group with CFS and early-life stress CFS was 'intermediate' after dexamethasone (Table 4). In our study, there were no significant differences in cortisol responses between the early-life stress CFS group and the control individuals, as clearly mentioned in the paper ${ }^{2}$ and in a related review article, published in $2007 .^{3}$

Hence, the findings of our group are difficult to reconcile with the hypothesis that hypothalamic-pituitary-adrenal axis hypofunction in CFS reflects a biological marker of early-life stress. Moreover, inversely to the findings by Heim et al. in patients with CFS, ${ }^{4}$ Weissbecker et al. ${ }^{5}$ found childhood trauma to be associated with an elevated cortisol response to awakening in fibromyalgia, a condition strongly related to CFS. Thus, further research is required to delineate the link between childhood trauma and hypothalamic-pituitary-adrenal axis dysfunction in CFS.
Department of Psychiatry (F. Van Den Eede), Department of Internal Medicine (G. Moorkens), Antwerp University Hospital, Wilrijkstraat 10, 2650 Edegem (Antwerp), Belgium. Correspondence to: F. Van Den Eede filip.van.den.eede@uza.be

\section{doi:10.1038/nrendo.2011.153-c1}

Competing interests

The authors declare no competing interests.

1. Papadopoulos, A. S. \& Cleare, A. J. Hypothalamic-pituitary-adrenal axis dysfunction in chronic fatigue syndrome. Nat. Rev. Endocrinol. 27, 22-32 (2012).

2. Van Den Eede, F. et al. Combined dexamethasone/corticotropin-releasing factor test in chronic fatigue syndrome. Psychol. Med. 38, 963-973 (2008).

3. Van Den Eede, F., Moorkens, G., Van Houdenhove, B., Cosyns, P. \& Claes, S. J. Hypothalamic-pituitary-adrenal axis function in chronic fatigue syndrome. Neuropsychobiology 55, 112-120 (2007).

4. Heim, C. et al. Childhood trauma and risk for chronic fatigue syndrome: association with neuroendocrine dysfunction. Arch. Gen. Psychiatry 66, 72-80 (2009).

5. Weissbecker, I., Floyd, A., Dedert, E., Salmon, P. \& Sephton, S. Childhood trauma and diurnal cortisol disruption in fibromyalgia syndrome. Psychoneuroendocrinology 31, 312-324 (2006). 